\title{
Impacto de la era del conocimiento y el big data en las empresas mundiales y ecuatorianas.
}

\author{
Fecha de recepción: 07/10/2019 • Fecha de aceptación: 03/12/2019 • Fecha de publicación:10/02/2020
}

Erika Jazmín Granizo Cuzco

Complejo Independiente del Valle

rikagranizo216@gmail.com

https://orcid.org/0000-0002-7286-5877

Abigail Lucía Ordoñez Jaramillo

abbyprincess1993@hotmail.com

https://orcid.org/0000-0002-7480-2044

\section{RESUMEN:}

El Big Data surge como resultado de la gran cantidad de información que se almacena como huellas tras realizar navegaciones en internet, los rastros que se dejan permiten que empresas con personas capacitas analicen estos datos y los usen de forma conveniente en distintos ámbitos encaminándose a realizar un mejor proceso de toma de decisiones que contribuyan a realizar mejores movimientos estratégicos. El propósito principal de este artículo es conocer el impacto de la herramienta Big Data como punto de impulso para el crecimiento empresarial a través del correcto proceso de toma decisiones basadas en el análisis de datos. Para ello se llevó a cabo una investigación de tipo empírico documental, mediante la revisión e investigación de fuentes bibliográficas y electrónicas, el mismo también es considerado como un enfoque cuantitativo ya que se analizan datos estadísticos. Los resultados a nivel mundial muestran que el Big Data es una herramienta que ha crecido de manera acelerada, la implementación de esta herramienta hasta el 2018 tiene un porcentaje del $11.24 \%$, y teniendo una proyección de 42 millones de ingresos hasta el 2018, y una expansión de esta herramienta hasta el 2018 de 6590.60 millones. A nivel nacional hay resultados positivos ya que de la encuesta realizada a 96 empresas el $72 \%$ indican que conocen esta herramienta al igual el $32.29 \%$ las empresas de Guayaquil la tienen implementada, en sí el Big Data es una herramienta que ayuda a la 
toma de decisiones y que poco a poco se ha ido involucrando en el mercado y dándole las oportunidades a las empresas a ser más competitivas.

PALABRAS CLAVE: proceso de toma de decisiones, movimientos estratégicos

\begin{abstract}
Big Data arises as a result of the large amount of information that is stored as footprints after browsing on the Internet, the traces that are left allow companies with trained people to analyze this data and use it conveniently in different areas, aiming to make a Better decision-making process that contributes to better strategic movements. The main purpose of this article is to know the impact of the Big Data tool as a momentum for business growth through the correct decision-making process based on data analysis. For this, an empirical documentary type investigation was carried out, through the review and investigation of bibliographic and electronic sources, it is also considered as a mixed approach since numerical data is used, that is, a quantitative method, and in turn qualitative An analysis of the previously investigated statistical data is performed. The results worldwide show that Big Data is a tool that has grown rapidly, the implementation of this tool until 2018 has a percentage of $11.24 \%$, and having a projection of 42 million revenue until 2018, and a expansion of this tool until 2018 of 6590.60 million. At the national level there are positive results because of the survey of 96 people, $72 \%$ indicate that they know this tool as $32.29 \%$ of the companies in Guayaquil have implemented this tool, if Big Data is a tool that helps to take of decisions and that little by little it has been getting involved in the market and giving companies the opportunities to be more competitive.
\end{abstract}

\title{
KEYWORDS: decision making process, strategic movements
}




\section{INTRODUCCIÓN}

La llegada del boom tecnológico desde la década de los 70 con la aparición de internet ha facilitado de una forma impresionante la conexión a larga distancia, así como también el acceso a enormes cantidades de información de todo tipo en corto tiempo, estos grandes bloques de información almacenados en la red día a día van en aumento dificultando el manejo adecuado por parte de las empresas, quienes deben encargarse de manejar datos referentes a clientes, producción, comercio, redes sociales entre otras. Todo este proceso de manejo se ve complicado analizándolo en conjunto, sin embrago al hablar del Big Data, herramienta cuya finalidad es facilitar la toma de decisiones basadas en análisis tras dividir la enorme cantidad de información en segmentos pequeños trasladados a distintos ordenadores por medio de algoritmos, técnicas estadísticas, herramientas matemáticas o análisis avanzados, se puede reducir el tiempo invertido y obtener grandes beneficios al saber manejar correctamente los resultados obtenidos.

El Big Data como herramienta empresarial puede ser muy útil en distintas áreas dependiendo las necesidades de las mismas, muchas empresas exitosas a nivel mundial han logrado aplicar el Big Data para conocer de mejor forma los requerimientos de sus clientes y brindar un mejor producto 0 servicio por medio de la información registradas en las bases de datos, expertos aseguran que la información que como usuarios dejamos en la red es usada para brindarnos una mejor experiencia en lo que estemos buscando, no es muy raro pensar que al realizar una búsqueda acerca de cualquier tema obtengamos inmediatamente sugerencias y publicidad relacionada, todo esto como resultado de las huellas que dejamos en el internet, a nivel empresarial funciona de la misma forma, las empresas buscan aplicar los beneficios del Big Data en distintos ámbitos del negocio como segmentar y fidelizar clientes, optimizar campañas de marketing, potenciar productos, analizar riesgos y poder tomas decisiones acertadas.

En la actualidad la mayoría de empresas mundiales han adoptado por aplicar la herramienta Big Data, la cual es utilizada para diferentes áreas, entre las más importantes está el área de Negocios, Marketing, Ventas y Talento Humano. Para el área de negocios la herramienta Big Data tiene la finalidad de estudiar el comportamiento de cada cliente, de esta manera llegarlos a conocer de tal forma en que se pueda hacer un análisis de las compras o facturaciones realizadas en un determinado tiempo, el Big Data ha creado una cadena de supermercados en donde se puede aplicar los análisis desarrollando de cada cliente ofreciendo así descuentos, promociones relacionadas a sus necesidades en los departamentos que frecuenten u obtengan más movimientos económicos. En el área de Talento Humano el Big Data ayuda a la toma de decisiones, permitiéndoles realizar una segmentación adecuada, facilitándoles la obtención de perfiles adecuados para el cumplimiento de los objetivos establecidos por la empresa.

A nivel del Ecuador existen en su mayoría organizaciones que no cuentan con esta herramienta, ya sea por su alto valor de implementación o debido a que las mismas no cuentan con una asesoría adecuada que les permita identificar y utilizar los beneficios y puntos estratégicos para adaptarlos a su modelo de negocio, radicando como el principal problema latente de abrirse a la era tecnológica que promete brindar grandes cambios a futuro. Tomando en cuenta este punto, este trabajo de investigación tiene como objetivo principal conocer el impacto de la herramienta Big 
Data como punto de impulso para el crecimiento empresarial a través del correcto proceso de toma decisiones basadas en el análisis de datos.

\section{BIG DATA}

La teoría del Big Data nace en el año 1997, los investigadores de la NASA, Michael Cox y David Ellsworth afirma que el gran aumento de datos se estaba convirtiendo en un problema para los sistemas informáticos actuales, Esto se da a conocer como el problema del Big Data los cuales se han ido mejorando, cada día la utilización del Big Data es más sencilla, y fácil de utilizarlo al igual en los últimos años su popularidad ha incrementado ya que se le identifica como una herramienta para la toma de decisiones para una organización. Los precios de instalación y almacenaje de datos son más económicos adaptándose a la necesidad de cada empresa, conforme pase el tiempo y avanza la tecnología esta herramienta sigue desarrollando técnicas estratégicas que permite incrementar el almacenamiento y gestión para los volúmenes de información que se presentaran en futuros años.

\section{MÉTODOS}

La presente investigación se plasma a través de un diseño empírico documental, cuya información se obtiene de fuentes bibliográficas y electrónicas, pretendiendo resaltar los puntos más relevantes de aplicar el Big Data como herramienta de crecimiento empresarial, basado en evidencias obtenidas a través del análisis y recopilación de información proveniente de distintas fuentes impresas y electrónicas pretendiendo obtener datos relevantes acerca del tema.

En este caso es un estudio con enfoque cuantitativo, puesto se analizan datos estadísticos, la información obtenida para el análisis ha sido extraída de revistas como Wikibon y Ticbeat que realizan investigaciones para publicar estadísticas mundiales referentes a distintos temas de interés y de un trabajo de tesis realizado por un estudiante de la Universidad de Guayaquil en el año 2018, el mismo que por medio de encuestas ha logrado obtener datos verídicos acerca del manejo del Big Data en el Ecuador, cabe recalcar que al ser un tema relativamente nuevo la información a nivel de país es un tanto escasa para realizar un análisis más profundo, sin embargo a través del presente estudio se pretende ampliar estos conocimientos mediante el manejo de un enfoque cuantitativo al analizar los datos extraídos y presentados a través de cuadros estadísticos, a fin de analizar los distintos puntos de vista de los nuevos usuarios de esta nueva herramienta tecnológica.

\section{RESULTADOS}

Según un estudio sobre ventas acerca del Big Data y su proyección para el 2018 realizado por Wikibon y Ticbeat que son comunidades profesionales dedicadas a resolver problemas de tecnología y negocios se presentan las siguientes graficas estadísticas. 
Estadísticas mundiales

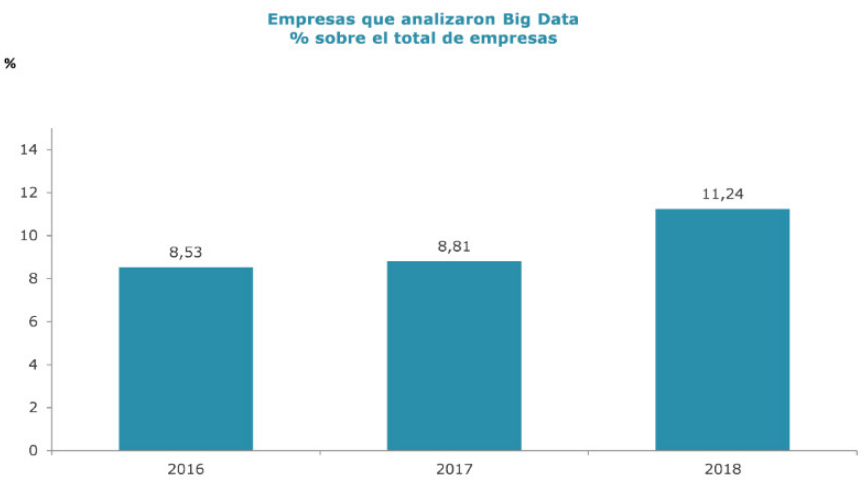

Figura 1.- Empresas que Implementan el Big Data. Fuente: (ticbeat.org)

Al observar la figura 1, se muestra el crecimiento en la implementación del Big Data en las empresas en los últimos 3 años, en donde para el año 2016 se observa un porcentaje de implementación del $8.53 \%$, en el siguiente año 2017 la implementación crece en un $0.21 \%$, es decir, que su implantación representa el 8,81\%, en el año 2018 su crecimiento aumenta en un $2.43 \%$ representando el $11,24 \%$ de la implementación de esta herramienta, como se puede observar cada año su implementación ha crecido lo que indica que cada vez más empresas se muestran receptivas a la llegada de la era tecnológica.

Forecast Revenue Big Data Market Worldwide 2011-2027

Big Data Market Size Revenue Forecast Worldwide From 2011 To 2027 (in billion U.S. dollars)

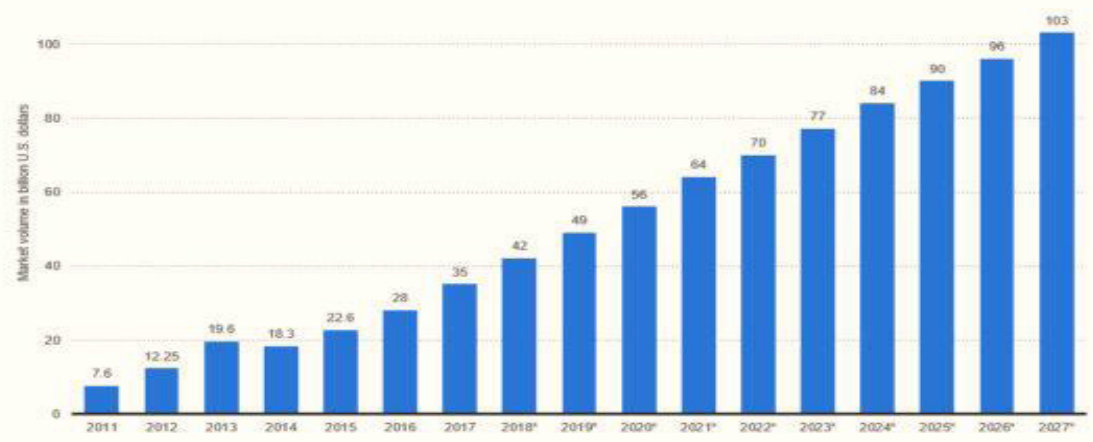

statista 5

Figura 2.- Proyección realizada por Ticbeat proyección de ingresos por Big Data para 2027.

Fuente: (ticbeat.org)

Al observar la figura 2, se puede identificar que la proyecciones realizadas del año 2011 al 2027, en cuanto a los ingresos percibidos por servicios en el mercado del Big Data aumenten de los 42.000 millones obtenidos en el 2018 a 103.000 millones para el 2027, dando a entender que 
supone un incrementos anual de $10,48 \%$, dicho crecimiento se ve refljado de forma notoria al compararlo con los 35.000 millones recaudados anteriormente frente a los 103.000 que se espera obtener en 9 años.

\section{America Latina}

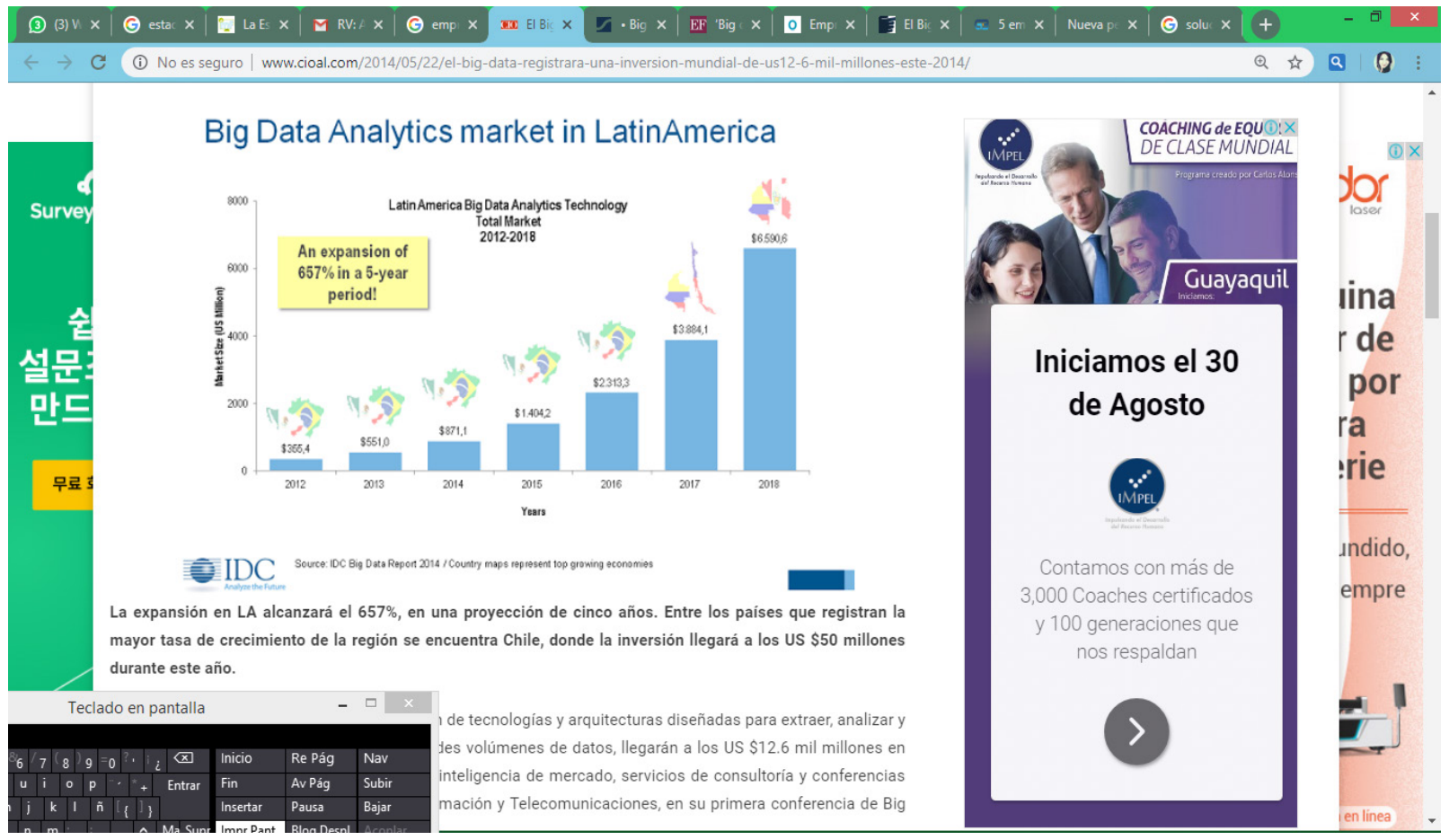

Figura 3.- Expansión de Big Data en América Latina. Fuente: (ticbeat.org)

Al observar la figura 3, se observa una proyección de la expansión del Big Data mostrando un crecimiento de $657 \%$ en 5 años, en donde uno de los paises con mayor tasa de crecimiento en la región es Chile, seguido por Colombia y Perú, dedibo a la alta y rapida adopción tecnologica al mostrase receptivos a incorporar estrategias de negocios, sumado a la creciente cantidad de proveedores del servicio y sus ofertas. Muchos de los otros paises latinoamericanos aun se encuentran en fase de descubrimiento, debido a que no entienden como opera y funciona el Big Data, recalcando el hecho de que solo cinco de diez compañias utilizan dicha herramienta y se encuentran mayormente en los paises que demuestran el mayor crecimiento.

\section{Estadísticas Nacionales (Guayaquil)}

En base a una encuesta realizada en la Ciudad de Guayaquil a una muestra de 96 empresas se extraen los siguientes datos: 


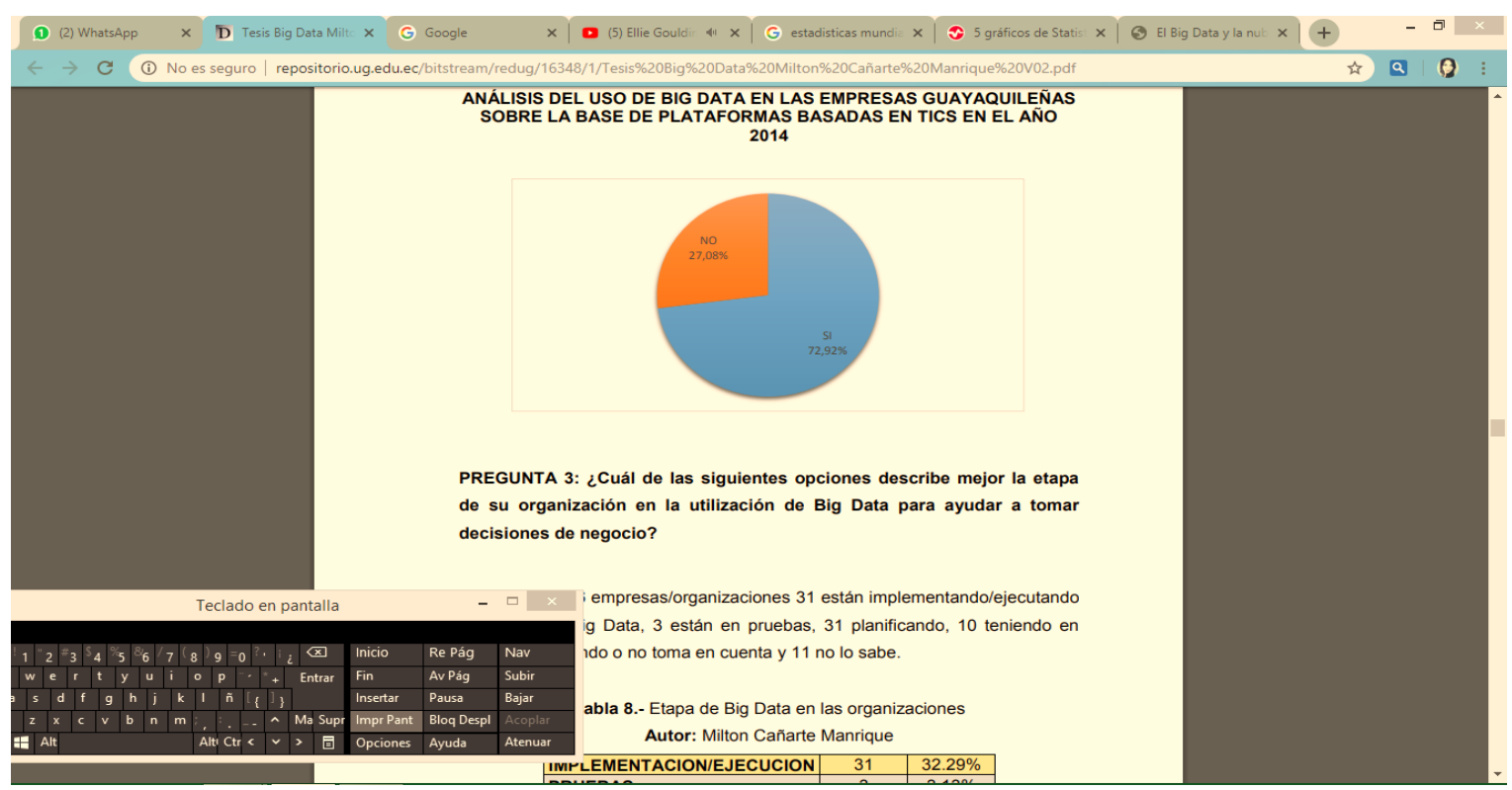

Figura 5.- Conoce acerca del Big Data. Fuente: (Repositorio Universidad de Guayaquil)

Al observar la figura 5, se puede deducir que el nivel de conocimiento en cuanto al Big Data entre los trabajadores de distintas empresas es bastante receptivo y que podría aumentar con ayuda de capacitaciones por parte de las distintas empresas que lo tengan implementado o lo deseen implementar

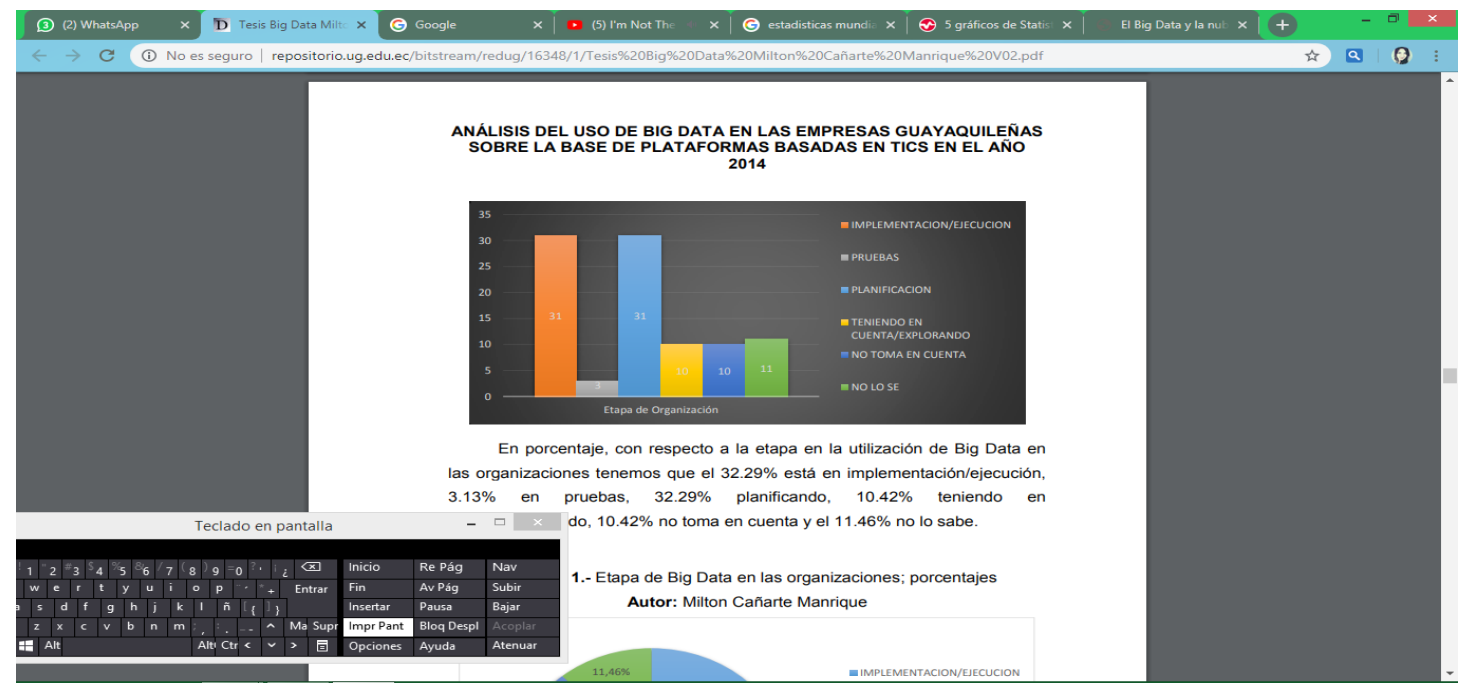

Figura 6. Implementación del Big Data. Fuente: (Repositorio Universidad de Guayaquil)

En la figura 6, se puede observar que en base a la muestra de 96 empresas u organizaciones encuestadas, 31 se encuentran implementando/ejecutando proyectos de Big Data, 3 están aplicando pruebas para su adaptación, 31 están planificando a futuro su implantación, 10 tienen en cuenta los beneficios que podrían obtener de esta herramienta o se encuentra aun explotando las posibilidades y 11 no conocen aun de esta herramienta. Hablando en porcentajes generales 
el $32,29 \%$ de las empresas de Guayaquil se encuentran en la fase implementación, el 3,13\% en pruebas, $32,29 \%$ planificando, $10,42 \%$ exploran y el $10,42 \%$ no lo conocen.

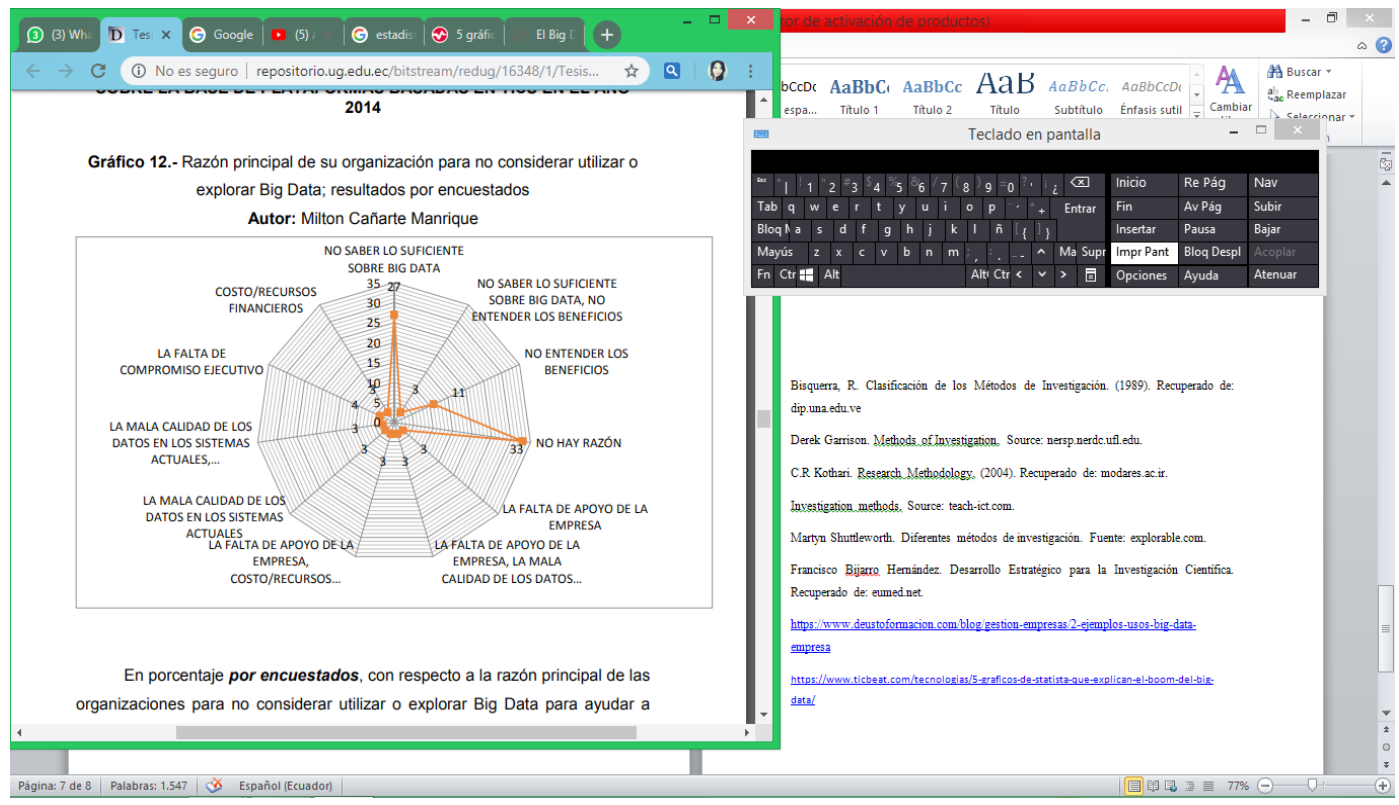

Figura 7. Razones para no Implementar el Big Data. Fuente: (Repositorio Universidad de Guayaquil)

En la figura 7, se puede apreciar que en su mayoría las razones principales para no considerar implementar el Big Data como herramienta de toma de decisiones en la organización se debe según 27 empresas a que no conocen lo suficiente sobre el tema, 11 no entienden cuáles son los beneficios y 33 no comprenden la razón de hacerlo.

En base a los datos y gráficos presentados se puede observar como el boom tecnológico ha contribuido al notable crecimiento económico de las empresas a nivel mundial proporcionando millones de ingresos a futuro, por el contrario, a nivel de país, en el Ecuador basándonos en la muestra obtenida, en su mayoría las empresas aún se encuentran tratando de adaptarse a estos cambios para obtener mayores beneficios e ir a la par con la innovación.

\section{DISCUSIÓN}

Al analizar los gráficos estadísticos, de las proyecciones e implementaciones de la herramienta Big Data se han podido mostrar tres escenarios, el escenario mundial, el escenario continental y el escenario a nivel de país, aunque la información recolectada no es tan profunda, si se puede señalar que frente al primer escenario el nivel de crecimiento en cuanto a la implementación del Big Data es mayor, debido a que principalmente países primermundistas como Estados Unidos y China lo manejan desde prácticamente su creación hasta el punto en que casi todas sus empresas lo mantienen actualmente, de ahí el porcentaje de crecimiento del $11 \%$ anual proyectado y reflejado en los 42.000 millones obtenidos en 2018 por este concepto, por otro lado al hablar a nivel de Latinoamérica el crecimiento en aplicación de esta herramienta muestra un porcentaje del $657 \%$ proyectado para 5 años, hecho que demuestra que el crecimiento es bastante acelerado 
debido a que recientemente países como Chile, Colombia y Perú se han abierto a adoptar nuevas tecnologías que les permitan competir en el mercado, a nivel de país Ecuador a igual que muchos otros como Bolivia y Venezuela aún se encuentra mayormente en la fase de descubrimiento de esta herramienta junto con otras más ligadas a la Era tecnológica, en donde solo 6 de cada 10 empresas conocen acerca del Big Data y solo 2 de estas 6 empresas aplican dicha herramienta o se encuentran en una fase de adaptación.

Muchos de los problemas ligados al poco impacto que tiene el Big Data en el Ecuador, se ven reflejados principalmente en la falta de conocimiento acerca del tema debido al poco desarrollo en cuanto a tecnologías que manejamos, lo poco receptivos que somos a abrirnos hacia nuevos conocimientos y no salir de nuestra zona de confort es lo que nos mantiene aún como países tercermundistas.

\section{CONCLUSIONES}

Al analizar el comportamiento del Big Data podemos decir que es una herramienta estratégica para las empresas, que ayuda al almacenamiento de datos en varias cantidades, al tener esta herramienta instalada en las empresas les ayuda a validar y analizar el comportamiento de los clientes y así como a tomar decisiones adecuadas tanto para mejorar sus productos o servicios, la implementación de esta herramienta se ha visto en continuo crecimiento demostrando hasta el 2018 un porcentaje de aumento del 11.24\%, y teniendo una proyección de 42 millones de ingresos a nivel mundial. A nivel nacional a pesar de que hay resultados positivos como se demuestra en la encuesta realizada a 96 empresas en la ciudad de Guayaquil, en donde el $72 \%$ indican que conocen esta herramienta, y que refleja a la vez que el $32.29 \%$ de estas empresas tienen implementado esta herramienta, aun no son números que se acerquen a la realidad en comparación con otros países como Chile, que se encuentran en el punto de auge frente a las nuevas tecnologías, teniendo en cuenta que el Big Data al ser una herramienta que ayuda a la toma de decisiones y que poco a poco se ha ido involucrando en el mercado dándole la oportunidades a las empresas a ser más competitivas, Ecuador debería ser más consiente de ir a la vanguardia de la innovación tecnológica. 


\section{REFERENCIAS BIBLIOGRÁFICAS}

Bisquerra, R. Clasificación de los Métodos de Investigación. (1989). Recuperado de: dip.una.edu.ve

Statista.com (2018). El Big Data y su Crecimiento. Recuperado de:

https://es.statista.com/estadisticas/624930/impacto-de-big-data-en-las-companias-a-nivel-mundial/

Ticbeat.com(2018). El Boom del Big Data. Recuperado de:

https://www.ticbeat.com/tecnologias/5-graficos-de-statista-que-explican-el-boom-del-big-data/

Wikibon. Org(2019). El Big Data y sus Proyecciones. Recuperado de: https://wikibon.com/research/big-data/

Cañate, M. (2014). Análisis del uso del Big Data en las empresas Guayaquileñas sobre la base de las Tics. Recuperado de:

http://repositorio.ug.edu.ec/bitstream/redug/16348/1/Tesis\%20Big\%20Data\%20Milton\%20Ca\%C3\%B1 arte\%20Manrique\%20V02.pdf 